La enseñanza de Historia en las profisiones de la Salud

\title{
La multitemporalidad como trabajo práctico: \\ una experiencia sobre la utilidad de la enseñanza de la Historia en la carrera de Trabajo Social
}

Canela Constanza Gavrila ${ }^{(a)}$

Andres Stagnaro(b)

Gavrila CC, Stagnaro A. Multitemporality as practical work: an experience about the usefulness of History teaching for the career of Social Work. Interface (Botucatu). 2018; 22(67):1017-28.

This article presents a pedagogical proposal for the framework for a history teaching to be part of the career of Social Work. The aim of this course is to shed light on the existence of different temporalities within the professional interventions of social workers. Because of the profession's link with the field of medicine, we question how this relationship is perceived by students, especially that between history and Social Work, in order to discover the best didactic strategies to construct historical knowledge.

Keywords: History. Social work. Professional education.
En el siguiente trabajo nos proponemos presentar una propuesta pedagógica, en el marco de la asignatura de Historia de la carrera de Trabajo Social, para la comprensión de la existencia de diferentes temporalidades en la intervención profesional de un/a trabajador/a social. Puesto que esta profesión estuvo fuertemente vinculada con la medicina, nos preguntamos, a fin de realizar un diagnóstico, de qué modo es percibida por los estudiantes esta relación, en particular entre la historia y el Trabajo Social, con el objetivo de avanzar en mejores estrategias didácticas en la construcción del conocimiento histórico.

Palabras clave: Historia. Trabajo social. Formación profesional.

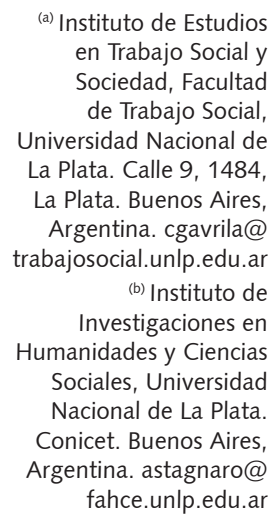

(a) Instituto de Estudios en Trabajo Social y Sociedad, Facultad de Trabajo Social, Universidad Nacional de La Plata. Calle 9, 1484, La Plata. Buenos Aires, Argentina.cgavrila@ trabajosocial.unlp.edu.ar (b) Instituto de Investigaciones en Humanidades y Ciencias Sociales, Universidad Nacional de La Plata. Conicet. Buenos Aires, Argentina. astagnaro@ fahce.unlp.edu.ar 


\section{Introducción}

El Trabajo Social (en adelante TS) se caracteriza por ser una profesión reconocida y legitimada en la sociedad por responder a las necesidades sociales en el proceso de producción y reproducción de sus medios de vida y de trabajo1. Su emergencia está ligada al fenómeno que Harold Perkin Ilamó la "sociedad profesional", en tanto su reconocimiento y legitimidad están vinculados a un proceso de conformación profesional que identifica una demanda determinada de la sociedad y se propone atenderlas a través de la jerarquización de saberes específicos avalados por el Estado mediante la expedición de títulos habilitantes.

Dado que el TS opera sobre sectores sociales incapaces de satisfacer sus necesidades básicas para la reproducción material, la intervención no está separada de las condiciones socio históricas del desarrollo capitalista, como tampoco de los mecanismos, dispositivos y reglas de juego que se imprimen en la toma de decisiones y modos de dominación del Estado en cada momento. Esta relación entre la intervención sobre la cuestión social y los modos históricos en que se configuraron las respuestas para distintas problemáticas de reproducción social hacen que el estudio de la historia y de las ciencias sociales cobren relevancia para vincular problemáticas actuales con un escenario histórico que lo antecede.

Sobre las contradicciones para la reproducción es que el TS proyecta posibles soluciones para la intervención que no resultan aisladas sino constitutivas del carácter ideológico que hace de algunas cuestiones problemáticas válidas o no de atender. Estas configuraciones ideológicas son el conjunto de normas, valores e ideales que realizados mediante ritos y rituales construyen ciertos arquetipos como normales y deseables, y objetan lo que no responda a dicho orden ${ }^{3}$. Las configuraciones ideológicas correspondientes a cada período particular en el desarrollo de las contradicciones entre el capital y el trabajo configuran variadas constantes ideológicas que definen el trabajo de los futuros profesionales de la asistencia

En este sentido la historia cobra relevancia al develar el carácter ideológico en que se construyeron los abordajes a la cuestión social. Esta íntima relación entre las transformaciones económicas y sociales con las posibles soluciones que el TS proyecta y realiza para la intervención sobre las refracciones de la cuestión social(c), no se traducen como un vínculo de utilidad para los estudiantes.

En este punto nos convocamos a reflexionar si el carácter accesorio y aislado con que la asignatura historia es considerada por los y las estudiantes para el desarrollo formativo no es parte de la vieja contradicción que guarda esta profesión en el campo de las ciencias sociales, donde la urgencia por la intervención en la realidad inmediata opera en desmedro de una reflexión situada en un tiempo de mayor alcance. Este problema manifiesta una concepción inmediatista de la intervención que olvida que el TS es un dispositivo estratégico en el abordaje de cuestiones de orden socio-histórico para el tratamiento de asuntos doméstico, y viceversa ${ }^{3}$. En este rumbo propondremos un abordaje crítico del sentido de lo "contemporáneo" para los estudiantes, avanzando en una conceptualización que permita comprender la contemporaneidad no solo en términos de lo intempestivo, sino en vinculación con otros tiempos, mediante un desfasaje que permita la percepción del presente sin esencialismos ${ }^{5}$.

En esta vinculación entre las problemáticas particulares y las generales no solo se esconde una preocupación por el tiempo histórico, sino también, por como estas prácticas reales y concretas, moldean distintas subjetividades que se insertan en relaciones sociales y colectivas. Es así que nuestra insistencia por establecer

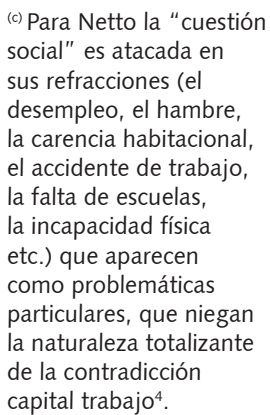


en las clases mayores diálogos entre la historia y el TS no resulta de un capricho disciplinar, sino de un interés por complejizar la multiplicidad del tiempo histórico que constituye a la cuestión social actual y a los sujetos que la componen. Esta motivación por repensar la historia y el estudio de la misma desde la formación en TS forma parte de un interés mayor por abordar críticamente el pasado de la disciplina, para desmitificar las apariencias humanitarias y universalizantes que desde la pretendida ingenuidad epistemológica e ideológica encubrieron el carácter conservador de la misma ${ }^{1}$.

Por esta razón nos proponemos realizar una reflexión acerca de los desafíos con los que nos encontramos al momento de abordar la historia social argentina y latinoamericana con estudiantes de TS. El trabajo presenta tres instancias: en principio una breve descripción de la materia en el contexto general de la formación en la actualidad, junto a una breve historización del ingreso de las ciencias sociales en la disciplina. En segunda instancia presentaremos las opiniones manifestadas por los estudiantes respecto a la pregunta ¿Por qué estudiar historia de Argentina y Latinoamérica en la formación en Trabajo Social? Por último, y acorde a los objetivos de este trabajo, realizaremos un diálogo entre las instancias anteriores de reflexión y de diagnóstico a fin de compartir una propuesta pedagógica plausible de ser utilizada como actividad de síntesis y evaluación final. Con ella pretendemos presentar fuentes históricas de primera y segunda mano, de distintos espacios y tiempos, a fin de dar cuenta de la historicidad de la cuestión social y su abordaje profesional.

\section{Los inicios profesionales de las agentes del Trabajo Social}

La situación política y social de principios del siglo XX en Argentina se caracterizó por varias tensiones entre ellas la de crecimiento económico, que estaba sujeto a una serie de vaivenes y no lograba superar recurrentes crisis, en un movimiento descripto como un "péndulo de la riqueza"6. Este no era solo de la riqueza sino que también atendía a los movimientos oscilantes del Estado en relación a sus principios constitutivos entre una pretendida imagen republicana y moderna y el avance genocida de la frontera ganadera de fines del siglo XIX, que favoreció un mayor despliegue del modelo agroexportador. Este modelo económico no implicó, como en otros casos latinoamericanos, la dualización total de la economía en un sector exportador y una economía de subsistencia, sino que favoreció el desarrollo de una incipiente industria con mano de obra extranjera llegada por inmigraciones masivas del período, generando un proceso de urbanización en toda la zona pampeana con eje en la ciudad de Buenos Aires y sus alrededores.

El proceso de modernización de la economía incluyó un doble proceso de industrialización y urbanización que fortalecieron la dependencia de los trabajadores de ingresos extra salariales que ya no podían suplir con productos de la economía doméstica. Esto generó el crecimiento de una masa de trabajadores y trabajadoras cuyos salarios no podían costear su reproducción, dando lugar a tensiones sociales que se suponían podía poner en peligro la cohesión social, como sostiene Robert Castel'7.

Dadas las condiciones precarias de desarrollo de estos sectores, debieron soportar epidemias que azotaron a la población local, fenómeno que promovió una serie de transformaciones institucionales, políticas y económicas. Junto con estas problemáticas, la disminución de la natalidad en los ámbitos urbanos resultaba alarmante. Como contrapartida, el Estado propuso distintas políticas maternalistas focalizadas ${ }^{8}$ en la consolidación de la familia, el desarrollo doméstico, la protección del binomio madrehijo y en la disminución de la mortalidad infantil ${ }^{9}$.

En este contexto, distintos sectores de la elite gobernante promovieron una intervención educativa y sanitaria, no solo de orden técnico, sino también moral ${ }^{10}$ con el objetivo de modificar hábitos de los sectores populares y así evitar la propagación de enfermedades ${ }^{(d)}$ Entre los agentes capaces de gestionar las soluciones requeridas para la asistencia social predominaron los médicos. 
La relación entre medicina y cuestión social iniciada a fines del siglo XIX como un acercamiento paliativo a las instalaciones urbanas, cobró otro carácter entrado el siglo XX. El discurso médico científico se ligó íntimamente en la planificación de políticas sociales y en la creación de redes entre instituciones de asistencia, prevención y moralización ${ }^{11}$.

En los casos de las grandes ciudades, como Buenos Aires y La Plata, el crecimiento urbano y poblacional hizo temer a los sectores gobernantes por el despliegue de la miseria, el hacinamiento y la expansión de epidemias. Estos hechos permitieron a la higiene acoplarse al proceso institucional suministrando un corpus normativo de orden prescriptivo y proscriptivo al Estado, e hizo de los médicos actores centrales en la proyección de las políticas sociales ${ }^{12}$.

El higienismo, como rama de la medicina, consideraba a la salud como una resultante de las condiciones física y social en que se desarrollaban los hombres y mujeres y para ello ejecutaban multiplicidad de estrategias educativas para intervenir sobre los sectores populares y facilitar la modificación de hábitos ${ }^{13}$.

En este escenario comenzó a funcionar la Escuela para Visitadoras de Higiene Social (EVHS) a cargo de la Cátedra de Higiene Médica y Preventiva de la Facultad de Ciencias Médicas (FCM) de la Universidad Nacional de Buenos Aires (UBA) en 1924 y en la Universidad Nacional de La Plata (UNLP) en el año 1938, antecedente universitario de la actual carrera de $\mathrm{TS}^{(\mathrm{e})}$. Estas instituciones formaron agentes auxiliares a los médicos, instruidas en conocimientos técnicos sobre higiene y prevención, que en comunión con sus dotes "amorosas y altruistas" 14 , pudieran intervenir con acciones de profilaxis, prevención social y educación sanitaria a fin de luchar contra la intemperancia de las costumbres perniciosas al individuo y la comunidad.

Para mitad del siglo XX la malla curricular de la carrera tuvo una serie de modificaciones vinculadas a las innovaciones en materia de políticas sociales y el alcance comunitario que pretendían obtener ${ }^{(f)}$. Si bien en un principio la formación alentaba a la subordinación con la ciencia médica, progresivamente el servicio social junto con la sociología, la administración sanitaria, la psicología, entre otras, se constituyeron en materias específicas de los planes de estudio, desequilibrando el peso de la palabra médica para la configuración de respuestas a la cuestión social a favor de una formación interdisciplinaria para pensar las refracciones de la cuestión social(g).

Entre las décadas de 1960 y 1970 la profesión experimentó cambios en consonancia con los sucesos políticos a nivel nacional y latinoamericano, como también de los pedidos de la Organización Mundial de Salud que nutrieron a la disciplina de nuevas concepciones teóricas y metodológicas relacionadas con la teoría de la dependencia y el desarrollismo. Ingresaron contenidos de Salud Pública y medicina comunitaria, junto con materias que permitirían complejizar la intervención sobre la cuestión social desde un abordaje de las ciencias sociales, como: psicología, antropología cultural y sociología. Es así que en el año 1974 se crea el título de Licenciado en Servicio Social, con una duración de cinco años ${ }^{(\mathrm{h})}$.

En la década de 1980 el proceso de normalización de las universidades nacionales pos dictadura permitió que el TS ganase autonomía como campo disciplinar. En la UNLP durante 1987 la carrera obtuvo la autonomía, desprendiéndose de la Facultad de Medicina al crearse la Escuela Superior de Trabajo Social. Aumentaron materias vinculadas al estudio de las problemáticas sociales, aspectos metodológicos y técnicos de intervención profesional. En el programa correspondiente al año 1989 las autoridades de la casa de estudios reconocieron la necesidad de abandonar la concepción paramédica del TS entendiendo que las problemáticas vinculadas al área de la salud eran sólo un

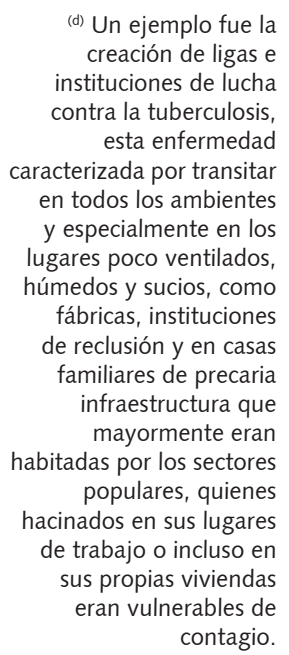
contagio.

(e) En el año 1930

otro grupo de

médicos higienistas

y reformadores crea

la Escuela de Servicio

Social en el Museo

Social Argentino, esta

pretendía la formación de profesionales que podían ser hombres o mujeres.

Los objetivos de esta

Escuela eran proporcionar enseñanza técnica y administrativa para jóvenes que pretendieran ocupar puesto de gestión en obras de asistencia y previsión social. Sin embargo esta experiencia no fue replicada en la ciudad La Plata dentro del ámbito universitario.

() Para esta reflexión tomamos como referencia la definición de currículo propuesta por De Alba en 1998

"se entiende a la síntesis de elementos culturales (conocimientos, valores, costumbres, creencias, hábitos) que conforman una propuesta político educativa pensada e impulsada por diversos grupos y sectores sociales cuyos intereses son diversos y contradictorios, aunque algunos tiendan a ser dominantes 0 hegemónicos, y otros tiendan a oponerse y resistirse a tal dominación o hegemonía"15 (p. 75) 
(g) "Las secuelas de la "cuestión social" son recortadas como problemáticas particulares (el desempleo, el hambre, la carencia habitacional, el accidente de trabajo, la falta de escuelas, la incapacidad física etc.) y así enfrentadas. La constatación de un sistema de nexos causales, cuando se impone a los intervinientes, alcanza a lo sumo un cuadro de integración de referencia centrado en la noción de integración social [...] Así, la "cuestión social" es atacada en sus refracciones, en sus secuelas aprehendidas como problemáticas cuya naturaleza totalizante, si es asumida consecuentemente, impediría la intervención." ${ }^{4}$ (p. 22).

(h) Para un análisis pormenorizado del proceso de modernización del Servicio Social en la ciudad de La Plata se sugiere la lectura de la Tesis de maestría de Néstor Arrúa disponible en http://sedici.unlp.edu. ar/handle/10915/52560.

(i) Ver: Plan de estudios de la carrera de Trabajo Social. 1989. Fundamentación pp. 3-4.

(j) Plan de estudios 2015. pp 4-7. exponente más -pero no exclusivo- de la problemática social, al igual que la pobreza, la marginalidad, el empleo, entre otras; que exigían un tratamiento desde las ciencias sociales ${ }^{(i)}$.

En la actualidad los y las trabajadoras sociales han ganado autonomía profesional, y han logrado multiplicar los espacios de inserción profesional, desde el área de justicia, educación, salud, minoridad, comunidades urbanas, rurales e indígenas, hasta intervenir en empresas, relaciones laborales, previsión y seguridad social, entre otras. Esta ampliación del campo profesional, manifiesta el carácter multifacético de la cuestión social y proyecta constantes desafíos para los y las futuros agentes en formación, hecho que exige un mayor acercamiento a las ciencias sociales.

\section{La Historia Social para estudiantes de Trabajo Social}

La asignatura Historia Social Argentina y Latinoamericana (HSAL) se inscribe en el primer año de la carrera de Trabajo Social perteneciente al Plan de Estudios vigente desde el año 2015. En este se reconoció la necesidad de que los estudiantes de TS tengan como base de su formación la "cuestión social" desde una fundamentación socio- histórica. Así la materia es la única dedicada, exclusivamente, a la historia social en el programa y por ello debe cubrir una secuencia temporal de dos siglos, desde la formación de los Estados nacionales y el ingreso en el mercado mundial, es decir, las últimas décadas del siglo XIX, hasta los debates sobre el cambio de época en Latinoamérica y las tensiones entre populismo y neodesarrollismo en la última década.

El reconocimiento de la centralidad de la cuestión social como base de la fundamentación socio histórica de la intervención en la realidad social, propone un análisis de tres actores fundamentales: el Estado, los sujetos sociales y sus necesidades, y por último, la trabajadora social con su saber específico. Con este paradigma se pretende una formación que permita comprender, interpretar y resignificar las particularidades de cada situación, a fin de reconocer el carácter ideológico de la profesión y, con ello, trascender el carácter inmediatista de la intervención. Se espera que los y las futuras profesionales puedan comprender el Estado y su relación con la sociedad, en particular con las problemáticas que se expresan de la conflictividad social y las políticas sociales como estrategias de intervención respecto de los mismos, para que puedanfortalecer los proyectos colectivos de resistencia y transformación social en que se involucren ${ }^{(j)}$.

Esta materia, además, se establece como punto de inicio del Trayecto Socio Histórico de la formación $n^{(k)}$. Siguen a esta Economía Política, Teoría del Estado, Teoría del Derecho y Derecho Social, Política Social y Conformación de la Estructura Social Argentina, de modo, que HSAL presenta un marco histórico sobre el que los y las estudiantes podrán articular y complejizar otros conocimientos del Plan de Estudios.

Como asignatura del trayecto inicial, y reconociendo la importancia del vínculo entre historia y TS, consideramos imprescindible incorporarestrategias pedagógicas interdisciplinarias que nos acerquen a trabajar sobre la construcción histórica de la estructura socioeconómica y política de nuestra sociedad actual, las constantes ideológicas que configuraron instituciones y modos de control social, las múltiples resistencias, la formación de proyectos alternativos, las configuraciones de lo cotidiano, entre otras cuestiones, que posibiliten a los y las estudiantes pensarse como sujetos históricos, que se hacen y rehacen en ese proceso, del que incluso salen prácticas renovadoras ${ }^{1}$. 
Uno de los desafíos inmediatos es valorizar el desarrollo y el análisis conceptual de los múltiples tiempos de la contemporaneidad ${ }^{5}$, el estudio de los sujetos y asociaciones posibles entre estos, el análisis de los distintos modos de intervención, el desarrollo de las instituciones de control social, como también los sujetos que intervinieron en la construcción de las políticas sociales, entre otras cuestiones, como estrategias posibles para acercar a los y las estudiantes a los análisis y estudios de ciencias sociales que trasciendan la primacía del orden "práctico" y coyuntural de su profesión.

Es importante destacar que el estudiantado del primer año se corresponde mayormente con recientes egresados y egresadas de la educación secundaria y provienen de provienen de distintas formaciones académicas, cuestión que hace imprescindible reconocerlas para poder trabajar sobre ellas en la instancia del trabajo práctico. Otro elemento característico del estudiantado en TS es su elevada matrícula femenina, que a pesar de no encontrase presentada en cifras oficiales desde la institución, resulta evidente cada vez que uno ingresa en las aulas de la facultad, cuestión que resulta del legado asistencial, auxiliar y subalterno de la disciplina ${ }^{16}$.

Ahora bien, todo este análisis acerca de los diálogos entre la historia y el TS, junto con la caracterización del estudiantado, no resulta suficiente a priori para la planificar las acciones a desarrollar con los grupos que anualmente se renuevan. Asumimos que nuestro rol como docentes de historia no solo debe facilitar la comprensión de los contenidos históricos prescriptos en el programa, sino también animar al desarrollo de la comprensión. De algún modo, enseñar historia es guiar otro modo de aprehensión de la realidad donde distinguir procesos, rupturas y continuidades de las estructuras sociales de las que son partícipes. Para ello, comprendemos que en el proceso de construcción del conocimiento, el estudiantado es un sujeto activo del mismo, que junto a los y las docentes, construyen las herramientas para que puedan fundar sus propios procedimientos de aprendizaje en función de esquemas previos que se reestructuran con las nuevas nociones adquiridas ${ }^{17}$.

Para dar paso a la acción con los y las estudiantes indagamos sobre que piensan de las utilidades de estudiar HSAL en la formación profesional en TS. Presentaremos algunas de estas respuestas para realizar una propuesta didáctica que permita trabajar sobre los supuestos de los y las estudiantes.

\section{¿Para qué sirve estudiar historia en Trabajo Social?}

En la primera clase del ciclo lectivo 2017 iniciamos el diálogo con esta pregunta ¿Para qué sirve estudiar historia en Trabajo Social? La respuesta era entregada por escrito y de manera anónima. Esperábamos encontrarnos con los preconceptos y expectativas que el estudiantado tenía sobre la materia y con ello obtener pistas que nos permitan trazar estrategias de trabajo. Con estos indicios pensamos como maniobra posible realizar una actividad de repaso e integración que detallaremos más adelante que favorezca la continuidad del estudiantado luego del primer exámen(l).

Si bien era un total de cincuenta y ocho estudiantes en la planilla, al ser la primera clase del año un grupo de ingresantes estaba ausente seguramente tratando de encontrar la comisión, el/ la docente y el aula correcta. Estaban presentes cuarenta y dos estudiantes que respondieron ¿Para qué sirve estudiar historia en Trabajo Social?

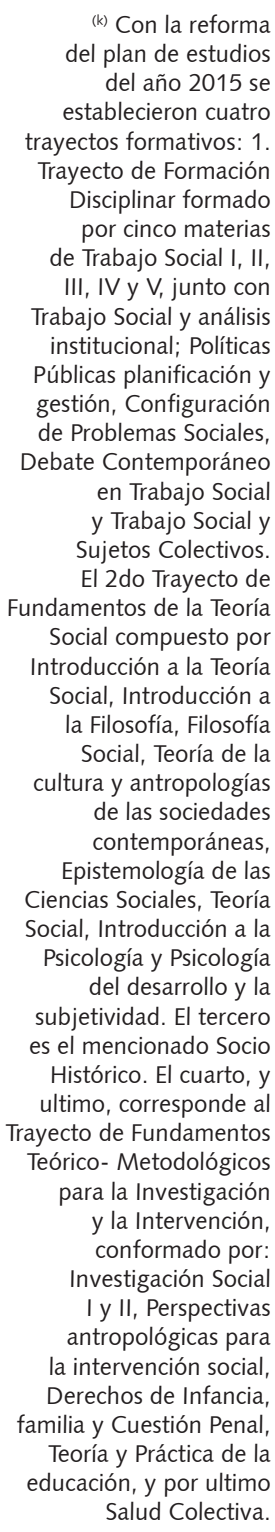

(k) Con la reforma del plan de estudios del año 2015 se establecieron cuatro trayectos formativos: 1 Trayecto de Formación Disciplinar formado por cinco materias de Trabajo Social I, II, III, IV y V, junto con Trabajo Social y análisis institucional; Políticas Públicas planificación y gestión, Configuración de Problemas Sociales,

Debate Contemporáneo en Trabajo Social y Trabajo Social y Sujetos Colectivos. El 2do Trayecto de Fundamentos de la Teoría Social compuesto por Introducción a la Teoría

Social, Introducción a la Filosofía, Filosofía Social, Teoría de la cultura y antropologías de las sociedades contemporáneas,

Epistemología de las

Ciencias Sociales, Teoría

Social, Introducción a la

Psicología y Psicología del desarrollo y la subjetividad. El tercero es el mencionado Socio Histórico. El cuarto, y ultimo, corresponde al

Trayecto de Fundamentos Teórico- Metodológicos para la Investigación y la Intervención, conformado por: Investigación Social I y II, Perspectivas antropológicas para la intervención social, Derechos de Infancia, familia y Cuestión Penal,

Teoría y Práctica de la educación, y por ultimo Salud Colectiva.

\footnotetext{
(I) Este primer examen que rinden los estudiantes aborda los contenidos trabajados durante todo el primer cuatrimestre que abarca desde 1860 hasta 1955. En muchos casos los y las estudiantes abandonan la materia antes de intentar rendirlo.
} 
Luego de leer las respuestas consideramos que estas podían agruparse en tres grandes núcleos, junto con algunas de las opiniones expresadas de manera anónima.

En principio encontramos una fuerte identificación de la historia como maestra de vida. Así lo enuncian dos estudiantes: "Estudiar historia sirve para tener conocimiento de los diferentes contextos históricos, porque para entender el hoy debemos conocer lo que pasó antes"; "La historia nos permite entender la cuestión social actual y a partir de experiencias anteriores no cometer los errores en el hoy".

Estas consideraciones - que aparecen de manera mayoritaria entre el estudiantado-identifican a la historia como un receptáculo de múltiples experiencias que tiene una función liberadora para los sujetos actuales en tanto ilumina el presente y el futuro para no repetir los errores del pasado ${ }^{18}$. El problema que guarda esta consideración es que el pasado parece no ser constitutivo de los sujetos, sino aislado y sin conexiones que inviten al estudiantado a identificarse como parte del proceso histórico, y por tanto, resulte dificultoso establecer un correlato entre la actualidad y el pasado, o identificar cambios, rupturas y continuidades.

Otra tipificación que apareció en las respuestas es que la HSAL es un escenario y telón de fondo para el desarrollo del TS. Quienes se acercaron a esta postura fue un grupo minoritario, aunque claros en la expectativa sobre la materia. Uno de ellos sostuvo "La historia sirve para conocer los procesos que transforman el contexto y los enfoques del trabajo social".

En este sentido, la historia abonaría al conocimiento de las razones por las cuales aún la profesión es necesaria para la sociedad, pero no parecería influir sobre los individuos, ni sobre sus condiciones de vida, intereses, ocupación, ideología y sexualidad. El problema que encubre esta consideración de la historia como accesoria del desarrollo profesional y aislada de los sujetos y sus problemáticas, es que no contempla como el carácter ideológico y subjetivo de las condiciones sociales produce efectos en la historia económica y política, como también en las relaciones afectivas ${ }^{3}$.

Un último sentido, sobre la relación entre historia y TS condensa y supera las nociones de magistra vitae y de historia como escenario inerte.

Al decir de un estudiante "Pienso que los problemas sociales en los que vamos a intervenir son construcciones sociales históricas, por eso es importante conocer lo sucedido en el pasado para entender el presente, viendo avances y retrocesos"

Aquí la historia es considerada como parte de las problemáticas y resoluciones que aborda el TS, donde se entienden las transformaciones políticas y sociales, desde la noción de conflicto y disputa en la que se configuran los sujetos sociales. La historia aparece como "materia misma de la intervención social" puesto que el TS trabaja sobre las configuraciones de esa historia y se sitúa en distintas coyunturas sociales ${ }^{3}$. Esto permite acercarnos a la idea de la historia como el haz de sombra que ilumina la contemporaneidad del momento presente en términos de Agamben ${ }^{5}$. Es decir, comprender como a partir de la operación de percibir, dividir e interpolar el tiempo, el sujeto está en condiciones de transformarlo -el presente de la acción de la intervención- y ponerlo en relación con otros tiempos más allá de aquél vivido. Ejercer, en última instancia, la capacidad de citar la historia ya no a su arbitrio, sino como una exigencia metodológica ineludible.

Esta es una concepción dinámica de la historia constituida por conflictos y disputas que colaboran en las transformaciones de la cuestión social, y a su vez influyen dialécticamente con la subjetividad de los futuros "usuarios" del TS.

En función de estas respuestas realizamos una propuesta didáctica que permitiera avanzar sobre las relaciones entre historia y TS, y evidenciara el carácter ideológico de la práctica profesional. El objetivo principal de la actividad es que los estudiantes se identifiquen como sujetos sociales capaces de intervenir en el entramado histórico que constituye la cuestión social y, además, realicen un ejercicio de percepción temporal que les permita reconocer la particularidad de las problemáticas sociales de un momento de la estructura social determinado, es decir, evitar el riesgo del inmediatismo que supone la primacía de la actualidad ahistorizada.

El ejercicio propuesto pretende que los estudiantes intenten resolver situaciones hipotéticas como trabajador social sumergido en las problemáticas de una época determinada y no como un sujeto externo y omnisciente, sino históricamente situado. 
Además, se promueve el proceso de diálogo entre distintos registros textuales (textos académicos y fuentes de época) y se favorece, así, el proceso de apropiación por parte de los estudiantes de los contenidos del programa de la materia. La presentación de los resultados del ejercicio obliga a los y las estudiantes a fomentar la presentación oral y escrita en forma clara en una instancia formativa fundamental que es la puesta en común. Este tipo de intervenciones se encuentran en sintonía con los objetivos pedagógicos declarados en el programa.

Otro objetivo pedagógico de la actividad es funcionar como hilo conductor de los distintos contenidos de la materia ya que la misma se ordena cronológicamente desde mediados del siglo XIX hasta principios del siglo XXI y se dicta a lo largo de todo un año académico.

Uno de los textos que más gusta entre los y las estudiantes es el de Andrea Oliva "Trabajo Social y lucha de clases. Análisis histórico de las modalidades de intervención en Argentina", específicamente los capítulos I "Demandas colectivas y respuestas institucionalizadas" y el capítulo II "Configuración de los modos de intervención", trabajado en las clases prácticas. Este texto es el único de la materia que aborda el proceso de institucionalización del TS desde la configuración del campo profesional de las visitadoras de higiene, y en ello atiende a distintas modalidades de intervención (como el suministro de recursos, visitas de profilaxis, de control de las condiciones de trabajo y de control de las condiciones de vida). Una de las cosas que genera aceptación es que los alumnos pueden identificar como la profesión inicia su recorrido para dar respuesta a la cuestión social, cómo se la define, y como en ese proceso se configuran distintos espacios de intervención y herramientas e instrumentos de trabajo profesional. Con el texto de Oliva a nuestro favor, es que nos planteamos elaborar una propuesta pedagógica para una clase de repaso y síntesis previa al primer examen.

La propuesta de trabajo propone que los y las estudiantes "ensayen" diagnósticos sobre distintas problemáticas de la cuestión social a principios del siglo XX y que para ello pongan en diálogo textos correspondientes a la cronología a trabajar. Con ese objetivo, ofreceremos tres fuentes documentales distintas, cada grupo trabajará con una. La primera es la "Memoria de la administración municipal de ciudad de La Plata" (1936), en ella se describen las acciones que realiza el Estado a través de sus agentes, los problemas de intervención y las estrategias para el abordaje. En la segunda fuente trabajamos con dos discursos del presidente Juan Domingo Perón (1946-1955) en los que se alude a las nuevas políticas sociales de su gobierno. Por último, sumamos un extracto de un discurso de Gabriela Laperriere, reconocida antecesora de las inspecciones sociales, donde denuncia el proyecto del Dr. Belisario Roldan, y en ello manifiesta los conocimientos que ella había producido como agente del servicio social.

\section{Propuesta para los y las estudiantes}

\section{Consignas de trabajo}

Ustedes trabajarán en grupos de no más de seis estudiantes y simularán ser un equipo profesional a cargo de diagnosticar la situación presentada por los docentes y proponer una modalidad de intervención. La situación deberá ser construida en base a las lecturas específicas del programa y las fuentes que se transcriben. Deberán atender a las condiciones sociales descriptas en los textos a fin de generar una escena históricamente situada. A partir del planteamiento de la escena realizarán sus observaciones y propuestas, con un formato similar a un diario de campo, y plantearán una posible estrategia de abordaje. Cada grupo compartirá en una instancia plenaria su propuesta.

A fin de facilitar la elaboración de su diagnóstico y propuesta les proponemos algunas preguntas que operarán como guías en su reflexión:

¿Cuáles son las problemáticas para la reproducción social que se detectan en el caso a trabajar? ¿En qué contexto histórico particular emergen estas situaciones? 
(m) "Usuarios" es como se denomina en lenguaje nativo a los sujetos beneficiarios de la acción
¿Qué sujetos sociales aparecen como beneficiarios de la intervención -"usuarios" (m)?

¿Qué sujetos profesionales podrían intervenir? ¿El grupo profesional que sugieren formar podría ser parte de una institución? ¿Cuál?

De acuerdo a la propuesta de Andrea Oliva ¿creen que sería pertinente utilizar alguno de los modos de intervención de los inicios de la profesión? De ser afirmativa su respuesta ¿cuál sería el indicado?

Justifiquen su diagnóstico y propuesta de intervención.

Memoria de la administración municipal. 1936. Ciudad de La Plata. pp 119- 120.

Inspección de Sanidad Jefe: Dr. Laureano Arau. Médicos: Dr. Eduardo Lavalle Dr. Julio Echagüe Dr. Florencio Alesso Dr. RaulStagnari

La municipalidad cuenta con un servicio sanitario cuya labor se desarrolla dentro del palacio, donde se hallan sus instalaciones, y fuera de él. En el edificio de la Comuna están: el Dispensario Antivenéreo; Laboratorios de análisis clínicos, industriales y bromatológicos; Gabinete Radiológico; Consultorio Ginecológico y otorrinolaringología.

La campaña sanitaria se cumple en toda la ciudad de acuerdo a las ordenanzas en vigor y cuyo detalle se observará enseguida.

El Consultorio Externo de la Inspección de Sanidad, que funciona en el Palacio Municipal realiza las siguientes funciones:

$1^{\circ}$ Asistencia médica gratuita a los pobres.

$2^{\circ}$ Examen médico de los conductores de vehículos, carros, automóviles, motocicletas etc. Este examen comprende: $1^{\circ}$ Examen clínico general.

$2^{\circ}$ Examen de la agudeza visual con optópicos. $3^{\circ}$ Examen de agudeza auditiva. $4^{\circ}$ Examen de sistema nervioso.

$3^{\circ}$ Examen de los empleados y obreros municipales. $1^{\circ}$ Riguroso examen médico de los aspirantes a empleos municipales en el que se constate las condiciones de salud al incorporarse a la administración comunal. $2^{\circ}$ Constatación de enfermedades del personal municipal a los efectos de justificar inasistencias. $3^{\circ}$ Informes periciales a los efectos de la ley de accidentes de trabajo ante el Departamento Provincial del Trabajo y de los Tribunales.

$4^{\circ}$ Dispensario Antivenéreo: A) Examen de prostitutas. $1^{\circ}$ Examen clínico general. $2^{\circ}$ Examen ginecológico. $3^{\circ}$ Examen de la piel. $4^{\circ}$ Análisis clínicos que comprenden reacciones de Wasserman y Kahn; esputos; flujo vaginal, uretral, etc. $5^{\circ}$ Examen radiológico. B) Examen de varones: $1^{\circ}$ Exámen clínico general. $2^{\circ}$ Examen de la piel. $3^{\circ}$ Análisis clínicos y examen genital.

Campaña Sanitaria $5^{\circ}$ Inspección Sanitaria de locales. $1^{\circ}$ Casa de vecindad, hoteles, fondas, pensiones, restaurantes, rotiserías, confiterías, bares etc. 
$6^{\circ}$ Inspección sanitaria de piletas de natación.

70 Desratización y desinfección de locales.

$8^{\circ}$ Inspección de prostíbulos.

$9^{\circ}$ Reconocimiento a domicilio: de empleados y obreros municipales a objeto de justificar su inasistencia.

$10^{\circ}$ Difusión de preceptos higiénicos: por medio de impresos para combatir enfermedades infecto- contagiosas más comunes. (Fuente histórica n¹)

Extracto del Discurso del coronel Perón sobre la política social del Estado transmitido por la red argentina de radiodifusión, Diciembre 2 de 1943

El Estado cumplirá ahora su deber social

[...] El Estado manteníase alejado de la población trabajadora. No regulaba las actividades sociales como era su deber, solo tomaba contacto en forma aislada cuando el temor de ver turbado el orden aparente de la calle lo obligaba de la calle le obligaba a descender de Latorre de marfil de su abstencionismo suicida. No se percataban los gobernantes de que la indiferencia adoptada ante las contiendas sociales, facilitaba la propagación de esta rebeldía porque era precisamente el olvido de los deberes patronales que, libres de la tutela estatal, sometían a los trabajadores a la única ley de su conveniencia. [...] Los problemas que sean consecuencia natural de los hechos sociales serán estudiados y recibirán la rápida solución que justicieramente merezcan [...]. (Fuente histórica $N^{\circ}$ 2)

Extracto del discurso En Berisso, 10 de agosto de 1944

[...] Hemos de organizar la acción social, ya sea en su aspecto de asistencia social como en el de previsión social. Entendemos por asistencia social el principio que asegura que el hombre debe tener su salario para comer, habitar y vestirse. Cualquier otro gasto que se produzca y que deba abonar el obrero, sea suprimido, a fin de que el Estado se haga cargo, ya sea enfermedad, o pasajera inepcia para el trabajo. Y cuando fuera de carácter permanente, por invalidez o vejez, el hombre debe ser tomado a cargo del Estado. (Fuente histórica № 2)

Extracto de "Dos iniciativas femeninas" Gabriela Laperriere. 1901. Revista antituberculosa. Tomado de Gabriela Laperriere de Coni. Tejero Coni, Graciela, Oliva, Andrea. 2016.

Es utopía pensar por el momento en medios prácticos para que la mujer abandone el trabajo fabril y se dedique al cuidado de su interior, esposo e hijos. Transcurrirá mucho tiempo antes de que modifique el estado actual y de que vislumbre para ella esta perspectiva. Solo sucederá cuando el salario del obrero sea elevado, para satisfacer las necesidades de su familia. Mientras falten mejoras fundamentales, sociales y económicas, no queda otro recurso que aliviar en lo posible su situación.

La mujer obrera debería cobrar en realidad dos salarios con los quehaceres domésticos que realiza. Debería pagar a otra mujer si por enfermedad o por exceso de trabajo en el taller no pudiera atender su casa. Se calcula desde las 4.30 hs en que se levanta para estar a las 6 en el taller, después de haber preparado el desayuno, arreglado su casa, vestido a sus hijos, etc... hasta las 9 de la noche, después de haber limpiado la vajilla, cosido, lavado, etc, que ha trabajado sin interrupción 17 horas.

¿Cuánto resistirá a este recargo, máxime cuando desempeña a veces en los talleres trabajos musculares que deberían ser reservados a hombres? ¿Cómo no será víctima de la tuberculosis si esta ataca principalmente a agotados y sin fuerzas para luchar? (Fuente histórica n³) 


\section{Consideraciones finales}

En este trabajo nos propusimos realizar una reflexión acerca de cómo enseñar historia en la carrera de Trabajo Social a partir de revisar las condiciones en que ingresaron las ciencias sociales en el desarrollo de la profesión. Sumamos a ello las consideraciones del estudiantado acerca de las utilidades de estudiar historia dentro de su formación. La reflexión de los estudiantes fue utilizada con una doble función: primero con un objetivo pedagógico exploratorio que desnaturaliza la presencia de la asignatura en el entramado curricular; y segundo, como un insumo fundamental que habilitase la reflexión sobre las formas en que la Historia se piensa como insumo fundamental para el desarrollo profesional de los trabajadores social.

En este sentido el diagnóstico llevó a considerar la creación de nuevas estrategias y actividades que permitiesen a los estudiantes a pensarse históricamente y concebirse como sujetos con capacidad para comprender diversas temporalidades y, a su vez, ser portadores de la capacidad de transformar dicha realidad. Esto implica resituar los distintos presentes de acción de la y el trabajador social. De esta forma el ejercicio propuesto permite trabajar sobre los significados de los diversos tiempos que componen un presente. Es decir, escapar de la tentación de la contemporaneización ahistórica permanente, entendida esta como la realidad actual que experimentan los estudiantes, al promover su resitualización en un contexto histórico diferente constituido de múltiples temporalidades.

A partir de estas reflexiones realizamos una propuesta pedagógica donde los vínculos entre historia y definición de problemáticas sociales de la cuestión social pudieran ser comprendidos desde el presente, siendo este el que otorga sentido, cuestiona y desnaturaliza al pasado. Si bien la propuesta aguarda ser puesta en práctica, esperamos con ella que los y las futuras profesionales puedan identificar las condiciones estructurales e históricas que hacen a las problemáticas individuales y colectivas sobre las que van a involucrar profesionalmente, e incluso puedan tener como insumo esa historia para pensar en las estrategias para intervenir.

\section{Colaboradores}

Canela Constanza Gavrila y Andres Stagnaro participaron en todas las fases de la producción del artículo y aprobaron la versión final presentada.

\section{Referencias}

1. Iamamoto M. Servicio social y división del trabajo. Un análisis crítico de sus fundamentos. San Pablo: Cortez; 1997.

2. Perkin H. The rise of professional society: England since 1880. London: Routledge; 2002.

3. Karsz S. Problematizar el trabajo social. Barcelona: GEDISA; 2007.

4. Netto JP. Capitalismo monopolista y cuestión social. San Pablo: Cortez; 2002.

5. Agamben G. Desnudez. Barcelona: Anagrama; 2011.

6. Rocchi F. Un largo camino a casa: empresarios, trabajadores e identidad industrial en Argentina, 1880-1930. En: Suriano J. La cuestión social en Argentina, 1870-1943. Buenos Aires: La Colmena; 2002.

7. Castel R. Las metamorfosis de la cuestión social. Una crónica del salariado. Buenos Aires: Paidós; 2009. 
8. Nari M. Políticas de maternidad y maternalismo político. Buenos Aires, 1890-1940. Buenos Aires: Biblos; 2004.

9. Biernat C, Ramacciotti K. Crecer y multiplicarse. La política sanitaria materno infantil. Argentina, 1900-1960. Buenos Aires: Biblos; 2013.

10. Ramacciotti K. La política sanitaria del peronismo. Buenos Aires: Biblos; 2009.

11. Armus D. El descubrimiento de la enfermedad como problema social. En: Lobato $M Z$. El progreso, la modernización y sus límites (1880-1916). Buenos Aires: Eudeba; 1999. p.132-54.

12. Vallejo G. Escenarios de la cultura científica argentina. Ciudad y Universidad (18821955). Madrid: Consejo Superior de Investigaciones Científicas; 2007.

13. Recalde H. La higiene y el trabajo. Buenos Aires: CEAL; 1988. (tomo 1).

14. Dezeo P. Discurso inaugural de la escuela para visitadoras de higiene social. Buenos Aires: Imprenta y casa Editora Coni; 1938.

15. De Alba A. Currículo, crisis y mito. Buenos Aires: Miño y Dávila; 1998.

16. Grassi E. La mujer y la profesión de asistente social. Buenos Aires: Humanitas; 1989.

17. Carretero M. Constructivismo y educación. Buenos Aires: Paidós; 1997.

18. Koselleck R. Futuro pasado. Para una semántica de los tiempos históricos. Barcelona: Paidós; 1993.

Gavrila CC, Stagnaro A. A multitemporalidade como trabalho prático: uma experiência sobre a utilidade do ensino de História na carreira de Trabalho Social. Interface (Botucatu). 2018; 22(67):1017-28.

No presente trabalho nos propomos apresentar uma proposta pedagógica para o ensino de História na carreira de Trabalho Social, para a compreensão da existência de diferentes temporalidades na intervenção profissional do trabalhador/a social. Posto que a profissão vinculou-se à Medicina, nos perguntamos sobre o modo que esta relação é percebida pelos estudantes, em particular a relação entre a História e o Trabalho Social, com objetivo de procurar melhores estratégias didáticas para a construção do conhecimento histórico.

Palavras-chave: História. Trabalho social. Formação profissional.

Sometido en 17/08/17. Aprobado en 21/06/18 